Published in Proteomics (2011) $11: 1798-1813$

\title{
Analysis of the xylem sap proteome of Brassica oleracea reveals a high content in secreted proteins
}

\author{
Laetitia Ligat $^{1,2 *}$, Emmanuelle Lauber $^{3 *}$, Cécile Albenne ${ }^{1,2}$, Hélène San Clemente ${ }^{1,2}$, \\ Benoît Valot ${ }^{4}$, Michel Zivy ${ }^{5}$, Rafael Pont-Lezica ${ }^{1,2}$, Matthieu Arlat ${ }^{3,6}$ and Elisabeth \\ Jamet ${ }^{1,2}$ \\ * These authors equally contributed to the work. \\ ${ }^{1 .}$ Université de Toulouse; UPS; UMR 5546, Surfaces Cellulaires et Signalisation chez les \\ Végétaux; BP 42617, F-31326 Castanet-Tolosan, France \\ 2. CNRS; UMR 5546; BP 42617, F-31326 Castanet-Tolosan, France \\ 3. Laboratoire des Interactions Plantes Microorganismes (LIPM); UMR CNRS-INRA 2594- \\ 441; F-31320 Castanet-Tolosan, France. \\ 4. CNRS, PAPPSO, UMR 0320 / UMR 8120 Génétique Végétale, F-91190 Gif sur Yvette, \\ France \\ 5. INRA, PAPPSO, UMR 0320 / UMR 8120 Génétique Végétale, F-91190 Gif sur Yvette, \\ France \\ ${ }^{6 .}$ Université de Toulouse; UPS; 118 route de Narbonne; F-31062 Toulouse, France.
}

Correspondence: Dr Elisabeth Jamet, UMR 5546 UPS/CNRS, Surfaces Cellulaires et Signalisation chez les Végétaux; BP 42617, F-31326 Castanet-Tolosan, France

E-mail: jamet@1rsv.ups-tlse.fr

Fax: +33(0)534323802

Keywords: Arabidopsis thaliana / Bioinformatics / Brassica oleracea / Cell wall protein / Glycoproteome / Xylem sap

Abbreviations: AGP, arabinogalactan protein; FLA, fasciclin AGP; GH, glycoside hydrolase; GRP, glycine-rich protein; LTP, lipid transfer protein; XTH, endotransglucosylase-hydrolase

\begin{abstract}
Xylem plays a major role in plant development, and is considered part of the apoplast. Here we studied the proteome of Brassica oleracea $\mathrm{cv}$ Bartolo and compared it to the plant cell wall proteome of another Brassicaceae, the model plant Arabidopsis thaliana. $B$. oleracea was chosen because it is technically difficult to harvest enough $A$. thaliana xylem sap for proteomic analysis. We studied the whole proteome and an $\mathrm{N}$-glycoproteome obtained after Concanavalin A affinity chromatography. Altogether, 189 proteins were identified by LC-MS/MS using Brassica EST and cDNA sequences. A predicted signal peptide was found in 164 proteins suggesting that most proteins of the xylem sap are secreted. Eighty one proteins were identified in the $\mathrm{N}$-glycoproteome, with 25 of them specific of this fraction, suggesting that they were concentrated during the chromatography step. All the protein families identified in this study were found in the cell wall proteomes. However proteases and oxidoreductases were more numerous in the xylem sap proteome, whereas enzyme inhibitors were rare. The origin of xylem sap proteins is discussed. All the experimental data including the MS/MS data were made available in the WallProtDB cell wall proteomic database.
\end{abstract}




\section{Introduction}

Xylem tissue is a major component of the vascular system of plants with a critical role in the transport of water, minerals and nutrients [1]. It is composed of tracheary elements, parenchyma cells, and fibers. During their differentiation, tracheary elements lose their nuclei and cellular content. A lignified secondary wall is formed. At the end of the process, cell death occurs, leaving a hollow tube which becomes a part of a vessel [2]. Xylem sap was shown to contain small molecular weight inorganic compounds and organic substances including hormones, amino acids, sugars, oligo- and polysaccharides, and proteins (for a review, see [3]). The question of the origin of xylem sap proteins was discussed and it was assumed that they could be breakdown products occurring during xylem formation or produced by parenchyma cells adjacent to xylem tissue $[3,4]$. Until now, only a few xylem sap proteins were shown to be synthesized in roots [5,6]. The current hypothesis is that proteins are secreted and/or synthesized by the stele cells and transported to the xylem vessels by the flow of water controlled by both transpiration in the leaves and pressure in the roots [3]. Then, the xylem sap is more and more considered as part of the apoplast [3].

Information on protein content of xylem sap is available for several plants such as Brassica napus [4, 7, 8], B. oleracea [7], Cucumis sativus [6, 7, 9], Cucurbita maxima [7], Glycine max [10, 11], Malus domestica [12], a poplar hybrid (Populus trichocarpa x P. deltoides) [13], Prunus persica [12], Pyrus communis [12], Solanum lycopersicum [14-16] and Zea mays [17]. Some of these studies were focused on some proteins, whereas others described xylem sap proteomes. However, because of the lack of genomic or EST sequences for all the plants studied, the identification of proteins by mass spectrometry (MS) and bioinformatics was mainly done using heterologous sequences. Several protein families were found such as glycosyl hydrolases (GHs) including xyloglucan endotransglucosylases-hydrolases (XTHs), peroxidases, proteases, lectins, pathogenesis-related proteins (PR-proteins), and cell wall structural proteins such as glycine-rich proteins (GRPs). It was also shown that the xylem sap proteome may change during plant-microbe interactions. Proteins homologous to basic glucanases (GH family 17, GH17) and PR4 were found in xylem sap of B. napus in response to infection by Verticillum longisporum and were assumed to contribute to defense [8]. Proteins homologous to cationic peroxidases and serine proteases were found to be induced in the soybean xylem sap in response to Phytophtora sojae elicitor and were related to programmed cell death [11]. After inoculation by the Bradyrhizobium japonicum symbiont, a XTH slightly accumulated in soybean xylem sap, but no function could be assigned to this 
protein [11]. On the contrary, a major Cys-rich protein of the healthy tomato xylem sap was shown to disappear after infection by Fusarium oxysporum [14].

A description of the xylem sap proteome of the model plant Arabidopsis thaliana should help us to better characterize this important compartment of the plant and might allow the characterization of determinants limiting vascular pathogen infection or facilitating their growth. However, such an analysis is still missing, probably because efficient xylem sap harvesting in A. thaliana is technically difficult. Such an analysis should complete proteomic studies on this model plant [18-27]. In this study, we took advantage of the close genetic vicinity between A. thaliana and another Brassicaceae, Brassica oleracea to identify proteins of the xylem sap. B. oleracea has two main advantages: (i) the diploid Brassica species are descended from an hexaploid ancestor and the genome of A. thaliana is similar to each of their hypothetical diploid progenitors and (ii) it is one $(2 \mathrm{n}=18, \mathrm{CC}$ genome) of the two ancestors of the $B$. napus amphidiploid (2n=38, AACC genome) [28] for which a systematic program of EST sequencing has been developed (http://compbio.dfci.harvard.edu/tgi/gi/bngi/GenInfo.html). Two different proteomes of $B$. oleracea xylem sap were studied: a xylem sap proteome and the xylem sap $N$-glycoproteome because $N$-glycosylation was assumed to be a major post-translational modification (PTM) that occurs in secreted proteins. The identified proteins are homologous to previously described cell wall proteins, except that no structural protein was identified [29]. The origin of xylem sap proteins is discussed using A. thaliana root transcriptomic data available online.

\section{Material and methods}

\subsection{Xylem sap harvesting}

Xylem sap harvesting method from the cultivated plant Bartolo cabbage (Brassica oleracea var. capitata) was adapted from [30]. Harvesting was found to be optimal for 6-8 week-old plants. Briefly, stems were cut with a razor blade $2-3 \mathrm{~cm}$ above the cotyledons and below the first leaves. Before sampling from the remaining stem on the root side, the cut surface was washed with water to remove the content of cut cells and the phloem sap which exudes after cutting, and gently dried with a paper towel. Aliquots of xylem sap was collected in a tube using a micropipette (Supporting information Fig. 1) and stored at $-20^{\circ} \mathrm{C}$ immediately after harvesting. All the xylem sap aliquots were pooled prior to further analysis. Before use, the samples were filtered using $0.45 \mu \mathrm{m}$ Millipore filters (Carrigtwohill, Ireland), to discard soil 
particles, microbial cells or tissue remnants. After 8 h-sampling, we obtained from 0.3 to 0.7 $\mathrm{mL}$ of xylem sap from one plant. The experiment was performed twice.

\subsection{Preparation of the protein samples for LC-MS/MS analysis}

After harvesting the B. oleracea xylem sap, the sample was dialyzed against buffer $1(20 \mathrm{mM}$ Tris- $\mathrm{HCl} \mathrm{pH} 7.4,150 \mathrm{mM} \mathrm{NaCl}, 1 \mathrm{mM} \mathrm{MgCl} / \mathrm{MnCl}_{2} / \mathrm{CaCl}_{2}$ ) in a Mega GeBAflex-tube (MWCO 12-14 kDa, GeBA, Yavne, Israel). Half of this sample, the "xylem sap proteome", was desalted using Econo-Pac 10 DG columns (Bio-Rad, Hercules, CA) and lyophilized. The second half of the sample used to get the "xylem sap $N$-glycoproteome" was directly separated by affinity chromatography on Concanavalin A (ConA) (Sigma, St Louis, MO). ConA lectin affinity chromatography is specific for Man residues and allows specific capture of $N$-glycoproteins [19]. Briefly, the resin was pre-washed with 20X volume of buffer 2 (20 $\mathrm{mM}$ Tris- $\mathrm{HCl} \mathrm{pH}$ 7.4, $1 \mathrm{M} \mathrm{NaCl}, 3.3 \mathrm{mM} \mathrm{MgCl} / \mathrm{MnCl}_{2} / \mathrm{CaCl}_{2}$ ) and equilibrated with $10 \mathrm{X}$ volume of buffer 1 . The dialyzed xylem sap $(10 \mathrm{~mL})$ was mixed with the matrix $(0.6 \mathrm{~mL})$ in batch for $1 \mathrm{~h}$ at $4^{\circ} \mathrm{C}$. After flow-through removal, the resin was washed three times with 1.5 $\mathrm{mL}$ of buffer 1 . Proteins were eluted with $3 \mathrm{X} 1.5 \mathrm{~mL}$ of buffer 1 supplemented with $1 \mathrm{M}$ methyl- $\alpha$-D-glucopyranose (Sigma). The first and second eluted fractions were combined and, after desalting using Econo-Pac 10 DG columns (Bio-Rad) and lyophilization, employed to get the xylem sap $N$-glycoproteome.

\subsection{Separation of proteins by SDS-PAGE}

The two protein samples (xylem sap proteome and xylem sap $N$-glycoproteome) were suspended in $300 \mu \mathrm{L}$ and $100 \mu \mathrm{L}$ of UHQ water respectively. Fifty $\mu \mathrm{L}$ of each sample were loaded on $10 \times 12 \times 0.15 \mathrm{~cm}$ SDS-polyacrylamide gels with a concentration of $12.50 \% /$ $0.33 \%$ of acrylamide/bisacrylamide. Separation was performed as previously described [31]. The gel staining was carried out with Coomassie Brilliant Blue (CBB) [32], silver nitrate [32], or with the $\beta$-glucosyl Yariv reagent [33]. The rest of the samples were dried under vacuum prior to LC-MS/MS analysis.

\subsection{LC-MS/MS analyses}

Prior to analysis, proteins were briefly separated by SDS-PAGE to get three samples in order to increase the efficiency of tryptic digestion. In-gel digestion was performed as previously described [34]. Separation of tryptic peptides was performed by HPLC on a NanoLC-Ultra system (Eksigent, Dublin, CA). A $4 \mu \mathrm{L}$ sample was loaded at $7.5 \mu \mathrm{L} \cdot \mathrm{min}^{-1}$ on a precolumn 
cartridge (stationary phase: C18 PepMap 100, $5 \mu \mathrm{m}$; column: $100 \mu \mathrm{m}$ inner diameter, $1 \mathrm{~cm}$ in length; Dionex, Voisins le Bretonneux, France) and desalted with 0.1\% HCOOH. After 3 min, the precolumn cartridge was connected to the separating PepMap C18 column (stationary phase: C18 PepMap 100, $3 \mu \mathrm{m}$; column: $75 \mu \mathrm{m}$ inner diameter, $150 \mathrm{~mm}$ in length; Dionex). Buffers were $0.1 \% \mathrm{HCOOH}$ in water (A) and $0.1 \% \mathrm{HCOOH}$ in $\mathrm{ACN}$ (B). The peptide separation was achieved with a linear gradient from 5 to $30 \%$ B for $28 \mathrm{~min}$ at $300 \mathrm{~nL} \cdot \mathrm{min}^{-1}$. One run took 45 min including the regeneration step at $95 \% \mathrm{~B}$ and the equilibration step at $95 \%$ A.

Eluted peptides were analyzed on-line with a LTQ XL ion trap (Thermo Electron, Thermo Fisher Scientific Inc, Courtaboeuf, France) using a nano electrospray interface as previously described with slight modifications detailed below [35]. Ionization (1.5 kV ionization potential) was performed with liquid junction and a noncoated capillary probe (10 $\mu \mathrm{m}$ inner diameter; New Objective). Peptide ions were analyzed using Xcalibur 2.07 (Thermo Fisher Scientific Inc) with the following data-dependent acquisition steps: (1) full MS scan (mass-tocharge ratio (m/z) 300 to 1400 , centroid mode); and (2) MS/MS (qz $=0.25$, activation time $=$ $30 \mathrm{~ms}$, and collision energy $=35 \%$; centroid mode). Step 2 was repeated for the three major ions detected in step 1. Dynamic exclusion was set to $30 \mathrm{~s}$.

A database search was performed with X! Tandem (version 2010.01.01.4) (http://www.thegpm.org/tandem/) using parameters and protein identification specifications previously described [35]. Three databases were used: (i) a Brassica napus EST database (Compbio, http://compbio.dfci.harvard.edu/tgi/cgi-bin/tgi/gimain.pl?gudb=oilseed_rape); (ii) the Arabidopsis Information Resource (TAIR, http://www.arabidopsis.org/) database (32825 entries, version 8); and (iii) a contaminant database (trypsin, keratins). To take redundancy into account, proteins with at least one peptide in common were grouped. Within each group, proteins with at least one specific peptide relatively to other members of the group were reported as sub-groups.

\subsection{Bioinformatics}

Two databases were used to analyze the cell wall proteome of B. oleracea: ProtAnnDB (http://www.polebio.scsv.ups-tlse.fr/ProtAnnDB/) for the annotation of A. thaliana proteins [36]; and WallProtDB which collects A. thaliana cell wall proteomes (http://www.polebio.scsv.ups-tlse.fr/WallProtDB/) [37].The Brassica proteins were annotated 
as previously described for A. thaliana proteins [36]. Several available software were used to predict sub-cellular localization and functional domains of proteins: TargetP (http://www.cbs.dtu.dk/services/TargetP/) and SignalP (http://www.cbs.dtu.dk/services/SignalP/) for sub-cellular localization and InterProScan for prediction of functional domains (http://www.ebi.ac.uk/Tools/InterProScan/). The results were combined to improve the quality of the predictions and to propose a structural and a functional annotation. The PROSITE software was used to predict $N$-glycosylation sites (http://www.expasy.org/prosite/). The AREX database was used to look for the root pattern of expression of $A$. thaliana genes homologous to those of $B$. oleracea encoding xylem sap proteins (http://www.arexdb.org/) [38].

\subsection{Implementation of MS/MS data in WallProtDB}

This WallProtDB knowledgebase was developed with PHP5/AJAX/MySQL5. It presently contains CWPs (476 from A. thaliana, 263 from Oryza sativa) and ESTs (162 from B. oleracea) which were classified as described [29]. B. oleracea sequences are linked to their closest homologues in $A$. thaliana as inferred from BLASTX searches (http://blast.ncbi.nlm.nih.gov/Blast.cgi, [39]). For B. oleracea, the proteomic data are linked to the MS data allowing protein identification. The spectra files are stored on a GPM server and usable with $\mathrm{X}$ ! Tandem via a simple hyperlink. WallProtDB can be queried via an html form with various criteria including plant species, organ, and experimental conditions. User can refine their selection if necessary and export the result in a tab delimited text, or export the sequences of interest in the FASTA format.

\section{Results}

\subsection{Harvesting of xylem sap and separation of proteins by SDS-PAGE}

The xylem was collected during $8 \mathrm{~h}$ from cut stems of B. oleracea (Supporting information Fig. 1). To avoid contamination by phloem sap and intracellular proteins of cut cells, the cut surface was rinsed with water. It was not possible to quantify the amount of proteins with the Bradford reagent probably because of their low concentration [40]. However, CBB stained bands were clearly visible after separation of proteins of the total extract (xylem sap proteome) by SDS-PAGE (Fig. 1, lane A). After lectin affinity chromatography on ConA to separate $N$-glycoproteins (xylem sap $N$-glycoproteome), a distinct profile of proteins was obtained showing enrichment in certain proteins and depletion in others (Fig. 1, lane B). Again, it was not possible to get reliable quantification of proteins after ConA affinity 
chromatography. In both cases, the profiles do not suggest any protein degradation. This was confirmed by a good distribution of the peptides allowing protein identification by LCMS/MS all over the protein amino acid sequences (Supporting information Tables S1 and S2). A staining with silver nitrate showed additional bands of lower molecular masses (Fig.1, lanes $\mathrm{C}$ and D). The total extract was also submitted to $\beta$-glucosyl Yariv reagent staining to reveal arabinogalactan proteins (AGPs) which are poorly stained by CBB and silver nitrate because of their high degree of glycosylation. A smear was observed at the top of the gel showing the presence of AGPs in the sample (Fig. 1, lane E). Both the total extract and the $\mathrm{N}$ glycoproteins retained on ConA were then analyzed by LC-MS/MS.

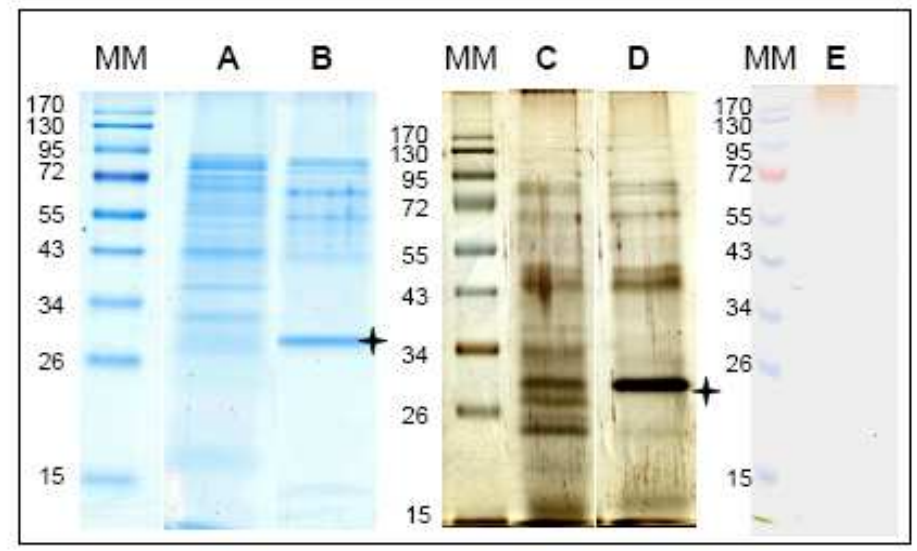

Figure 1. Separation of xylem sap proteins by SDS-PAGE.

Proteins from the xylem sap were separated by SDS-PAGE either directly after sampling and dialysis (lanes $\mathrm{A}$ and $\mathrm{C}$, xylem sap proteome), or after an additional step of affinity chromatography on ConA (lanes B and D, xylem sap $\mathrm{N}$-glycoproteome). The gel was stained with CBB (lanes A and B) or silver nitrate (lanes $C$ and $D$ ). Bands shown by a star correspond to the ConA protein leaking from the column. The total extract was also stained by the $\beta$-glucosyl Yariv reagent (lane E). MM are molecular mass markers $(\mathrm{kDa})$.

\subsection{Identification by LC-MS/MS of proteins present in the xylem sap proteome and in the xylem sap $\mathrm{N}$-glycoproteome trapped on ConA}

Most of the previous studies on xylem sap relied on separation of proteins by 2Delectrophoresis prior to MS analysis. However, 2D-electrophoresis can be limiting in the case of secreted proteins which are mostly basic glycoproteins [41]. In our study, proteins were not separated before digestion and liquid chromatography tandem MS (LC-MS/MS). This technique also allowed working with limiting amount of proteins. B. napus cDNA and EST sequences were used for proteins identification. In all cases, it was possible to identify 
proteins using Brassica EST or cDNA sequences. This was a great advantage since it allowed precise identification of the genes encoding the proteins especially in multigene families.

Altogether, 189 proteins were identified by LC-MS/MS with at least two peptides sequenced per protein (Table I, Supporting information Tables S1 and S2). One hundred and sixty four proteins were identified in the xylem sap proteome, whereas 81 proteins were identified in the xylem sap $N$-glycoproteome (Supporting information Table S3). Fifty six proteins were common to both proteomes. Twenty five proteins were only found in the xylem sap $\mathrm{N}$ glycoproteome. This fraction was probably enriched in these proteins after selection by the ConA affinity chromatography. Identification of proteins in previously published B. napus and B. oleracea xylem sap proteomes were done by comparison to heterologous sequences, mainly from $A$. thaliana $[4,7,8]$. We performed a new TBLASTN analysis against $B$. napus ESTs with the peptide sequences and obtained the identification of 45 different proteins. Then it was possible to compare these results to our data. Twenty out of these 45 proteins were also found in our study (see Table I). On the contrary, 25 proteins present in one of these proteomes were not found in ours probably because of different culture conditions. 
Table I. Proteins identified by LC-MS/MS in the xylem sap of B. oleracea

\begin{tabular}{|c|c|c|c|c|}
\hline $\begin{array}{l}\text { protein } \\
\text { number }\end{array}$ & $\begin{array}{c}\text { B. napus EST, cDNA } \\
\text { or protein accession } \\
\text { number }\end{array}$ & $\begin{array}{c}\text { homologue } \\
\text { in } A . \\
\text { thaliana } \\
\end{array}$ & predicted functional domain $^{c}$ & predicted sub-cellular localization $^{d}$ \\
\hline \multicolumn{5}{|c|}{ Proteins acting on carbohydrates (48) } \\
\hline 71 & TC38976 & At3g18080 & $\begin{array}{l}\text { GH1 (B-glucosidase, B-mannosidase) (AtBGLU44 } \\
\text { homologue) }\end{array}$ & chloroplast: 0.265 / SignalP-NN: 1-26, 0.679 \\
\hline $125-1$ & TC20974/TC45939 & At5g44640 & GH1 (B-glucosidase) (AtBGLU3 homologue) & N-ter missing (secretory pathway: 1-22, 0.811) \\
\hline $125-2$ & TC50854 & At2g44450 & GH1 (B-glucosidase) (AtBGLU15 homologue) & N-ter missing (secretory pathway: $1-22,0.853)$ \\
\hline $19-1$ & TC36871 & At5g64570 & GH3 (B-D-xylosidase) (AtXYL4 homologue) & N-ter missing (secretory pathway: $1-38,0.773$ ) \\
\hline $19-2$ & TC20792/CD814082 & At5g64570 & GH3 (B-D-xylosidase) (AtXYL4 homologue) & mitochondrion: 0.706 / SignalP-NN: 1-33, 0.797 \\
\hline 40 & TC20222/EE464798 & At5g64570 & GH3 (B-D-xylosidase) (AtXYL4 homologue) & chloroplast: 0.698 / SignalP-NN: $1-44,0.644$ \\
\hline 83 & TC32271 & At5g10560 & GH3 (B-D-xylosidase) & N-ter missing (secretory pathway: $1-18,0.311)$ \\
\hline 102 & TC49601 & At4g33820 & $\mathrm{GH} 10$ & N-ter missing (secretory pathway: $1-23,0.966)$ \\
\hline 23 & TC32833 & At4g15210 & $\mathrm{GH} 14$ & secretory pathway: $1-32,0.977$ \\
\hline $52-1$ & TC52964 & At4g25810 & $\begin{array}{l}\text { GH16 (xyloglucan:xyloglucosyltransferase) (AtXTH23 } \\
\text { homologue) }\end{array}$ & N-ter missing (secretory pathway: $1-24,0.988$ ) \\
\hline $52-2$ & TC23545 & At4g30270 & $\begin{array}{l}\text { GH16 (xyloglucan:xyloglucosyltransferase) (AtXTH24 } \\
\text { homologue) }\end{array}$ & secretory pathway: 1-22, 0.971 \\
\hline 3-1 & TC38745 (B. juncea) $[4,8]$ & At3g57240 & GH17 (ß-1,3-glucanase) & secretory pathway: 1-34, 0.960 \\
\hline $3-2$ & Q2HZ53 (B. juncea) & At3g57240 & GH17 (ß-1,3-glucanase) & N-ter missing (secretory pathway: $1-33,0.980)$ \\
\hline $47-1$ & TC26578 & At5g42720 & GH17 (ß-1,3-glucanase) & secretory pathway: $1-22,0.911$ \\
\hline $47-2$ & TC24955 & At4g34480 & GH17 (ß-1,3-glucanase) & secretory pathway: 1-22, 0.942 \\
\hline 66 & TC32141 & At4g34480 & GH17 (ß-1,3-glucanase) & secretory pathway: $1-22,0.931$ \\
\hline 95 & TC30288 & At5g55180 & GH17 (ß-1,3-glucanase) & secretory pathway: 1-22, 0.829 \\
\hline 128 & Q2VT22 (B. rapa) & At3g57260 & GH17 (ß-1,3-glucanase) & secretory pathway: $1-37,0.713$ \\
\hline 159 & TC49994 & At3g55430 & GH17 (ß-1,3-glucanase) & N-ter missing (secretory pathway: $1-28,0.973$ ) \\
\hline 28 & TC17043 & At5g24090 & GH18 (chitinase) & secretory pathway: $1-27,0.985$ \\
\hline 88 & TC54505 & At4g19810 & GH18 (chitinase) & N-ter missing (secretory pathway: $1-24,0.944)$ \\
\hline 8 & TC58115 [4] & At2g43590 & GH19 (chitinase) & secretory pathway: $1-33,0.938$ \\
\hline 110 & TC15913 & At3g12500 & GH19 (chitinase) & secretory pathway: $1-20,0.989$ \\
\hline 116 & TC25879 & At3g55260 & GH20 (N-acetyl-hexosaminidase) & N-ter missing (secretory pathway: $1-20,0.805)$ \\
\hline 56 & TC37303 & At3g56310 & $\mathrm{GH} 27$ & secretory pathway: $1-23,0.689$ \\
\hline 144 & EV101247 & At5g08370 & GH27 & N-ter missing (secretory pathway: $1-25,0.909)$ \\
\hline 26-1 & TC25741/TC46151 [4] & At3g61490 & GH28 (polygalacturonase) & N-ter missing (secretory pathway: $1-23,0.765)$ \\
\hline
\end{tabular}




\begin{tabular}{ll}
$\mathbf{2 6 - 2}$ & EV178995 \\
$\mathbf{2 6 - 3}$ & EV121625 \\
$\mathbf{2 7}$ & TC23229 \\
$\mathbf{8 1 - 1}$ & TC21367 \\
$\mathbf{8 1 - 2}$ & TC28593 \\
$\mathbf{1 0 7}$ & EE453748 \\
$\mathbf{9 7}$ & TC60884 \\
$\mathbf{6 5}$ & TC31486/TC29838 \\
$\mathbf{3 5}$ & TC47393 \\
$\mathbf{1 1 2}$ & TC17346 \\
$\mathbf{2 - 1}$ & TC43276 \\
$\mathbf{2 - 2}$ & TC43393 \\
$\mathbf{7 - 1}$ & TC53916 \\
& \\
$\mathbf{7 - 2}$ & TC61736 \\
$\mathbf{2 5}$ & TC31356 \\
$\mathbf{1 2 2}$ & TC41425 \\
$\mathbf{1 4 5}$ & AY036606 \\
$\mathbf{1 3 6}$ & CD814593 \\
$\mathbf{9 1}$ & TC16095 \\
$\mathbf{5 1}$ & TC16080 \\
$\mathbf{1 3 8}$ & DY023690 \\
\hline
\end{tabular}

At3g61490 GH28 (polygalacturonase)

At3g23500 GH28 (polygalacturonase)

At4g23500 GH28 (polygalacturonase)

At5g49215 GH28 (polygalacturonase)

At3g62110 GH28 (polygalacturonase)

At5g41870 GH28 (polygalacturonase)

At5g11720 GH31 ( $\alpha$-glucosidase) (AtGLU1 homologue)

At2g28470 GH35 (B-D-galactosidase) (AtBGAL8 homologue)

At5g13980 GH38 (a-D-mannosidase)

At5g13980 GH38 (a-D-mannosidase)

At3g 10740

At3g10740

GH51 ( $\alpha$-L-arabinofuranosidase/B-D-xylosidase) (AtASD 1 homologue)

At3g10740 GH51 ( $\alpha$-L-arabinofuranosidase/B-D-xylosidase) (AtASD homologue)

At3g10740 GH51 (a-L-

GH51 ( $\alpha$-L-arabi

homologue)

At5g34940 GH79 (B-D-glucuronidase)

At5g09760 pectin methylesterase (CE8)

At2g47550 pectin methylesterase (CE8)

At3g04910 pectin acylesterase (CE17)

At5g45280 pectin acylesterase (CE23)

At4g17030 expansin-like B (AtEXLB1 homologue)

At5g02260 alpha-expansin (AtEXPA9)
$\mathrm{N}$-ter missing (secretory pathway: 1-23, 0.765) $\mathrm{N}$-ter missing (secretory pathway: 1-22, 0.948) secretory pathway: $1-25,0.613$

$\mathrm{N}$-ter missing (secretory pathway: 1-21, 0.825) $\mathrm{N}$-ter missing (secretory pathway: 1-21, 0.946) $\mathrm{N}$-ter missing (secretory pathway: 1-21, 0.989) $\mathrm{N}$ ter missing (secretory pathway: 1-23, 0.976) secretory pathway: $1-23,0.991$

secretory pathway: $1-23,0.955$

$\mathrm{N}$-ter missing (secretory pathway: 1-21, 0.953 )

$\mathrm{N}$-ter missing (secretory pathway: 1-33, 0.962)

$\mathrm{N}$-ter missing (secretory pathway: 1-33, 0.962)

$\mathrm{N}$-ter missing (secretory pathway: 1-33, 0.962) secretory pathway: 1-33, 0.943 secretory pathway: $1-21,0.988$ $\mathrm{N}$-ter missing (SignalP-NN: 1-20, 0.803) secretory pathway: $1-23,0.860$ $\mathrm{N}$-ter missing (SignalP-NN: $1-44,0.417)$ secretory pathway: 1-23, 0.989 secretory pathway: 1-24, 0.988 $\mathrm{N}$-ter missing (secretory pathway: 1-21, 0.949)

\section{Oxido-reductases (39)}

\begin{tabular}{ll}
\hline $\mathbf{5 8}$ & TC47947 \\
$\mathbf{9 9}$ & TC33540 \\
$\mathbf{6 4}$ & TC25610 \\
$\mathbf{4 3}$ & TC36205 [4, 8] \\
$\mathbf{2 0 - 1}$ & TC30566 \\
$\mathbf{2 0 - 2}$ & TC27727 \\
$\mathbf{2 0 - 3}$ & TC25612 \\
$\mathbf{2 0 - 4}$ & NP1447930 \\
$\mathbf{7 7}$ & TC18276 \\
$\mathbf{4 9 - 1}$ & TC58934 [4] \\
$\mathbf{4 9 - 2}$ & TC61099 [4] \\
$\mathbf{1 3 - 1}$ & TC35223 [7]
\end{tabular}

At1g05260 peroxidase (AtPrx03 homologue)

At1g71695 peroxidase (AtPrx12 homologue)

At2g22420 peroxidase (AtPrx17 homologue)

At2g37130 peroxidase (AtPrx21 homologue)

At4g08770 peroxidase (AtPrx37 homologue)

At3g32980 peroxidase (AtPrx32 homologue)

At3g49120 peroxidase (AtPrx34 homologue)

At3g49120 peroxidase (AtPrx34 homologue)

At4g11290 peroxidase (AtPrx39 homologue)

At4g33420 peroxidase (AtPrx47 homologue)

At4g33420 peroxidase (AtPrx47 homologue)

At5g05340 peroxidase (AtPrx52 homologue) secretory pathway: $1-24,0.970$

secretory pathway: $1-31,0.856$

secretory pathway: $1-20,0.762$

secretory pathway: $1-29,0.981$

secretory pathway: $1-29,0.514$

chloroplast: 1-54, 0.309/SignalP-NN: 1-30, 0.684 secretory pathway: $1-32,0.820$

secretory pathway: $1-31,0.525$

secretory pathway: $1-23,0.962$

secretory pathway: $1-30,0.772$

secretory pathway: $1-25,0.920$

secretory pathway: 1-21, 0.796 


\begin{tabular}{ll}
$\mathbf{1 3 - 2}$ & TC45523 [4] \\
$\mathbf{1 3 - 3}$ & P00434 (B. rapa) \\
$\mathbf{1 1 - 1}$ & TC55805 [4] \\
$\mathbf{1 1 - 2}$ & EE424445 [7] \\
$\mathbf{1 1 - 3}$ & TC16466 \\
$\mathbf{5 9}$ & TC48654 \\
$\mathbf{1 1 1}$ & TC16200 \\
$\mathbf{2 2 - 1}$ & TC16404 \\
$\mathbf{2 2 - 2}$ & TC38876 \\
$\mathbf{2 2 - 3}$ & TC37557 \\
$\mathbf{2 2 - 4}$ & TC19179 \\
$60-1$ & TC39082 \\
$60-2$ & TC20750 \\
$\mathbf{1 1 7}$ & TC26850 \\
$\mathbf{3 8}$ & TC29181 \\
$\mathbf{3 9 - 1}$ & TC32771 \\
$\mathbf{3 9 - 2}$ & TC47186 \\
$63-1$ & TC21582 \\
$63-2$ & TC17946 \\
$63-3$ & TC23721 \\
$\mathbf{1 3 0}$ & TC21566 \\
$\mathbf{1 5 3}$ & TC25008 \\
$\mathbf{5}$ & TC18996 \\
$\mathbf{1 5 - 1}$ & TC17262 \\
$\mathbf{1 5 - 2}$ & TC38473 \\
$\mathbf{1 4 2}$ & ES903641 \\
$\mathbf{1 4 3}$ & EV087794 \\
& \\
\hline
\end{tabular}

At5g05340 peroxidase (AtPrx52 homologue)

At5g05340 peroxidase (AtPrx52 homologue)

At5g19890 peroxidase (AtPrx59 homologue)

At5g39580 peroxidase (AtPrx62 homologue)

At5g64120 peroxidase (AtPrx71 homologue)

At4g12420 multicopper oxidase (AtSKU5 homologue)

At4g12420 multicopper oxidase (AtSKU5 homologue)

At1g41830 multicopper oxidase (AtSKS6 homologue)

At1g76160 multicopper oxidase (AtSKS5 homologue)

At1g76160 multicopper oxidase (AtSKS5 homologue)

At1g76160 multicopper oxidase (AtSKS5 homologue)

At1g72230 uclacyanin (AtUCC8 homologue)

At1g72230 uclacyanin (AtUCC8 homologue)

At2g02850 plantacyanin (AtPNC homologue)

At2g25060 plastocyanin (AtEN7 homologue)

At4g27520 plastocyanin (AtEN12 homologue)

At4g27520 plastocyanin (AtEN12 homologue)

At4g31840 plastocyanin (AtEN13 homologue)

At4g31840 plastocyanin (AtEN13 homologue)

At4g31840 plastocyanin (AtEN13 homologue)

At5g15350 plastocyanin (AtEN22 homologue)

At5g15350 plastocyanin (AtEN22 homologue)

At4g20840 berberine-bridge oxido-reductase

At1g30760 berberine-bridge oxido-reductase

At2g34790 berberine-bridge oxido-reductase

At4g20840 berberine-bridge oxido-reductase

At5g44390 berberine-bridge oxido-reductase
$\mathrm{N}$-term missing (secretory pathway: 1-20, 0.916) $\mathrm{N}$-term missing (secretory pathway: 1-20, 0.916) secretory pathway: 1-25, 0.739 secretory pathway: 1-22, 0.962 secretory pathway: 1-23, 0.719

$\mathrm{N}$-ter missing (secretory pathway: 1-20, 0.989) secretory pathway: 1-20, 0.990 secretory pathway: $1-24,0.970$ secretory pathway: 1-23, 0.991

$\mathrm{N}$-ter missing (secretory pathway: 1-23, 0.984) secretory pathway: 1-23, 0.989 secretory pathway: 1-25, 0.972 secretory pathway: 1-24, 0.985 secretory pathway: 1-33, 0.825 secretory pathway: 1-23, 0.988 secretory pathway: 1-27, 0.975 secretory pathway: 1-28, 0.967 secretory pathway: 1-22, 0.989 secretory pathway: 1-21, 0.986 secretory pathway: 1-25, 0.991 secretory pathway: $1-26,0.970$ secretory pathway: $1-24,0.981$ secretory pathway: 1-26, 0.872 secretory pathway: 1-29, 0.979

$\mathrm{N}$-ter missing (secretory pathway: 1-26, 0.989) secretory pathway: 1-23, 0.803

$\mathrm{N}$-ter missing (secretory pathway: $1-30,0.825$ )

\section{Proteases (28)}

\begin{tabular}{|c|c|c|c|}
\hline $1-1$ & $\begin{array}{l}\text { TC18683/TC28749/ } \\
\text { TC22282 [4] }\end{array}$ & At5g67360 & Ser protease (ATSBT1.7, ARA12 homologue) \\
\hline $1-2$ & TC19435 & At5g67360 & Ser protease (ATSBT1.7, ARA12 homologue) \\
\hline $12 / 33$ & $\begin{array}{l}\text { ES901600/TC56831/ } \\
\text { TC46249 [4] }\end{array}$ & At1g20160 & Ser protease (ATSBT5.2 homologue) \\
\hline 29-1 & TC27270 & At4g36195 & Ser carboxypeptidase \\
\hline $29-2$ & TC25039 & At4g36190 & Ser carboxypeptidase \\
\hline 75 & TC27534 & At4g12910 & Ser carboxypeptidase (AtSCPL20 homologue \\
\hline $30-1$ & TC34104 & At4g30810 & Ser carboxypeptidase (AtSCPL29 homologue \\
\hline $30-2$ & TC47262 & At4g30810 & Ser carboxypeptidase (AtSCPL29 homologue \\
\hline
\end{tabular}

\section{secretory pathway: 1-29, 0.977}

$\mathrm{N}$-ter missing (secretory pathway: 1-24, 0.973) secretory pathway: 1-29, 0.991

secretory pathway: 1-21, 0.704 secretory pathway: $1-25,0.595$ secretory pathway: 1-23, 0.988 secretory pathway: 1-27, 0.992 secretory pathway: $1-25,0.984$ 


$\begin{array}{ll}\mathbf{6 9} & \text { EV124833 [7] } \\ \mathbf{7 4} & \text { EV124923 } \\ \mathbf{1 4} & \text { TC45864 } \\ \mathbf{6 2} & \text { TC23586/EV048732 } \\ \mathbf{1 8 - 1} & \text { TC21318 } \\ \mathbf{1 8 - 2} & \text { Q8LK82 } \\ \mathbf{1 8 - 3} & \text { ES904551 } \\ \mathbf{1 8 - 4} & \text { EE486131 } \\ \mathbf{4 4} & \text { EV196557/TC46757 } \\ \mathbf{9 3 - 1} & \text { TC21632 } \\ \mathbf{9 3 - 2} & \text { TC23324 } \\ \mathbf{9 3 - 3} & \text { TC33329 } \\ \mathbf{1 0 0} & \text { TC17031 } \\ \mathbf{1 1 5} & \text { TC23722 } \\ \mathbf{1 2 0} & \text { TC33693 } \\ \mathbf{9 6 - 1} & \text { B1Q3A2 } \\ \mathbf{9 6 - 2} & \text { TC59035 } \\ \mathbf{1 0 4} & \text { TC53234 } \\ \mathbf{1 1 9} & \text { TC33308 [4] } \\ \mathbf{1 3 4} & \text { TC26010 }\end{array}$

At1g11080 Ser carboxypeptidase (AtSCPL31 homologue)

At1g11080 Ser carboxypeptidase (AtSCPL31 homologue)

At3g45010 Ser carboxypeptidase (AtSCPL48 homologue)

At2g27920 Ser carboxypeptidase (AtSCPL51 homologue)

At5g10770 Asp protease

At5g10770 Asp protease

At5g10770 Asp protease

At5g10770 Asp protease

At5g10770 Asp protease

At3g18490 Asp protease

At3g18490 Asp protease

At3g18490 Asp protease

At5g07030 Asp protease

At3g54400 Asp protease

At1g79720 Asp protease

At1g47128 Cys protease (RESPONSIVE TO DEHYDRATION, RD21

homologue)

At5g43060 Cys protease

At5g60360

Cys protease (ALEURAIN LIKE PROTEASE, AALP

homologue)

At4g35350 Cys protease (XYLEM CYSTEINE PEPTIDASE 1, XCP1 homologue)

At4g01610 Cys protease
N-ter missing (secretory pathway: 1-30, 0.902) $\mathrm{N}$-ter missing (secretory pathway: $1-30,0.902$ ) secretory pathway: $1-25,0.603$ secretory pathway: 1-20 0.810

$\mathrm{N}$-ter missing (secretory pathway: 1-25, 0.276)

$\mathrm{N}$-ter missing (secretory pathway: 1-25, 0.276)

$\mathrm{N}$-ter missing (secretory pathway: $1-25,0.276$ )

$\mathrm{N}$-ter missing (secretory pathway: $1-25,0.276$ )

$\mathrm{N}$-ter missing (secretory pathway: 1-25, 0.276)

N-ter missing (SignalP-NN: 1-24, 0.895)

chloroplast: 0.342 / SignalP-NN: 1-24, 0.833

secretory pathway: 1-26, 0.456

secretory pathway: $1-19,0.805$

secretory pathway: 1-19, 0.294

secretory pathway: 1-28, 0.967

$\mathrm{N}$-ter missing (secretory pathway: 1-21, 0.993)

secretory pathway: 1-20, 0.987

secretory pathway: 1-22, 0.976

secretory pathway: 1-29, 0.986

secretory pathway: 1-29, 0.979

\section{Lipid metabolism (8)}

TC36034 [7]

132 TC19947

At2g44300 lipid transfer protein

At5G05960 lipid transfer protein

$\begin{array}{lll}\text { O82582 (B. oleracea) } & \text { At2g38540 lipid transfer protein (LTP1) } \\ \text { Q959F9 } & \text { At2g38530 lipid transfer protein (LTP2) }\end{array}$

68 Q9S9F9

$73 \quad$ EG019134

At5g59310 lipid transfer protein (LTP4)

DY024942

At1g09390

lipase acylhydrolase (GDSL family)

glycerophosphoryl diester phosphodiesterase

At4g26690 (MORPHOGENESIS OF ROOT 5, MHR5 homologue)

(GPI-anchored protein)

secretory pathway: 1-23, 0.936

secretory pathway: 1-23, 0.937

secretory pathway: $1-25,0.720$

secretory pathway: $1-25,0.970$

$\mathrm{N}$-ter missing (secretory pathway: 1-23, 0.967)

$\mathrm{N}$-ter missing (secretory pathway: 1-23, 0.976)

secretory pathway: 1-27, 0.983

N-ter missing (SignalP-NN : 1-27, 0.761)

$160 \quad$ TC50035

\section{Signaling (8)}

16-1 TC35372

16-2 TC31355

At5g55730 FLA (AtFLA1 homologue)

secretory pathway: 1-26, 0.799

At5g55730 FLA (AtFLA1 homologue)

secretory pathway: $1-26,0.799$ 


$\begin{array}{llll}\mathbf{4 6} & \text { TC39357 } & \text { At4g12730 } & \text { FLA (AtFLA2 homologue) } \\ \mathbf{3 7} & \text { TC21253 } & \text { At2g04780 } & \text { FLA (AtFLA7 homologue) } \\ \mathbf{2 4 - 1} & \text { TC21238 } & \text { At2g45470 } & \text { FLA (AtFLA8 homologue) } \\ \mathbf{2 4 - 2} & \text { TC30763 } & \text { At3g60900 } & \text { FLA (AtFLA10 homologue) } \\ \mathbf{6 1 - 1} & \text { TC32307 } & \text { At1g03870 } & \text { FLA (AtFLA9 homologue) } \\ \mathbf{6 1 - 2} & \text { TC23584 } & \text { At1g03870 } & \text { FLA (AtFLA9 homologue) } \\ \mathbf{4 5} & \text { TC26664 } & \text { At5g03170 } & \text { FLA (AtFLA11 homologue) }\end{array}$

secretory pathway: 1-23, 0.817 secretory pathway: $1-23,0.882$

$\mathrm{N}$-ter missing (secretory pathway: 1-25, 0.936)

$\mathrm{N}$-ter missing (secretory pathway: 1-25, 0.869)

secretory pathway: $1-25,0.912$

secretory pathway: $1-25,0.912$

secretory pathway: 1-24, 0.815

Interacting domains (8)

54

ES901495

113 TC20389 [4]

17 TC40783

$53 \quad$ TC32327 [4]

67-105 TC53636 [4]

123 TC41889

114 TC22255

At1g17860 protease inhibitor

At1g73260 protease inhibitor I3 (Kunitz legume)

At1g47960 pectinesterase inhibitor (PMEI)

At1g03220 xylanase inhibitor I (EDGP)

At1g78850 lectin (curculin-like, mannose binding)

At1g78830 lectin (curculin-like, mannose binding)

At1g26450 unknown function (X8 domain)

At1g21880 unknown function (LysM domain)

N-ter missing (secretory pathway: 1-19, 0.910

$\mathrm{N}$-ter missing (secretory pathway: 1-28, 0.985)

secretory pathway: 1-20, 0.974

secretory pathway: $1-23,0.917$

secretory pathway: 1-22, 0.990

secretory pathway: 1-22, 0.962

secretory pathway: 1-19, 0.809

secretory pathway: 1-27, 0.775

Miscellaneous (14)

TC59434

146 TC16085

$42 \quad$ TC32617

72 TC62786

10-1 TC21902

10-2 TC50714

55 TC25081

82 TC28594

86 TC38503 [4]

106 TC55340

126 TC54095 [4]

41-1 TC22018

TC22018

$\begin{array}{ll}41-2 & \text { TC22953 } \\ 154 & \text { TC33600 }\end{array}$

$\begin{array}{ll}\text { At4g23690 } & \text { dirigent protein } \\ \text { At1g13900 } & \text { purple acid phosphatase (AtPAP2 homologue) }\end{array}$

At3g07130 purple acid phosphatase (AtPAP15 homologue)

At3g07130 purple acid phosphatase (AtPAP15 homologue)

At5g34850 purple acid phosphatase (AtPAP26 homologue)

At5g34850 purple acid phosphatase (AtPAP26 homologue)

At3g19320 thaumatin

At2g14610 cysteine-rich secretory protein (SCP) (PR1 homologue)

At4g34180 cyclase

At3g62020 germin (GLP10 homologue)

At4g11650 osmotin (AtOSM34 homologue)

At2g02990 ribonuclease T2 (AtRNS1 homologue)

At1g26820 ribonuclease T2 (AtRNS3 homologue)

At1g78680 homologous to gamma-glutamyl hydrolase secretory pathway: 1-29, 0.994

$\mathrm{N}$-ter missing (secretory pathway: 1-19, 0.983)

$\mathrm{N}$-ter missing (secretory pathway: 1-19, 0.859)

$\mathrm{N}$-ter missing (secretory pathway: 1-19, 0.859)

secretory pathway: 1-27, 0.991

secretory pathway: 1-22, 0.772

secretory pathway: $1-26,0.974$

secretory pathway: 1-26, 0.798

secretory pathway: 1-23, 0.922

$\mathrm{N}$-ter missing (secretory pathway: 1-21, 0.970)

secretory pathway: 1-26, 0.991

secretory pathway: 1-26, 0.995

secretory pathway: 1-19, 0.983

secretory pathway: 1-24, 0.672

Unknown function (10)

$4 \quad$ TC27548

At5g48540 unknown function (DUF26)

secretory pathway: 1-20, 0.930

32

EV115188

At3g22060 unknown function (DUF26)

secretory pathway: 1-27, 0.880 


$\begin{array}{ll}\mathbf{4 8} & \text { TC55540 } \\ \text { 6-1 } & \text { TC30155 } \\ \mathbf{6 - 2} & \text { TC41195 } \\ \mathbf{6 - 3} & \text { TC28652 } \\ \mathbf{5 0 / 1 0 9} & \text { TC57337/TC59437/ } \\ \mathbf{1 0 3} & \text { EV164447 } \\ \mathbf{1 4 7} & \text { TC50570 } \\ \mathbf{1 5 0} & \text { TC16416 } \\ \end{array}$

\section{Intracellular (25)}

\begin{tabular}{ll}
\hline $9-1$ & TC23761 \\
$9-2$ & TC20982 \\
31 & Y09437 \\
34 & TC16377 \\
70 & TC16864 \\
76 & TC17072 \\
$78-1$ & TC18919 \\
$78-2$ & TC26676 \\
$79-1$ & TC17406 \\
$79-2$ & TC18964 \\
80 & TC20514 \\
84 & Q14U56 (B. oleracea) \\
$85-1$ & TC46191 \\
$85-2$ & EE426154 \\
92 & TC18282 \\
94 & TC28096 \\
101 & TC49144 \\
118 & TC31117 \\
124 & TC42226 \\
133 & TC22085 \\
137 & DW999965 \\
148 & TC17883 \\
149 & TC18121 \\
152 & TC20640 \\
156 & TC37476
\end{tabular}

At1g07390 unknown function (DUF568) (AIR12, AUXIN-INDUCED IN N-ter missing (SignalP-NN: 1-46, 0.260) ROOT CULTURES 12) (GPI-anchored protein)

At5g11420 unknown function (DUF642)

At5g11420 unknown function (DUF642)

At5g25460 unknown function (DUF642)

At5g12950 unknown function (DUF1680)

At2g15220 unknown function (basic secretory protein)

At1g76020 unnown function (thioredoxin fold)

At3g06035 unknown function secretory pathway: 1-22, 0.978

$\mathrm{N}$-ter missing (secretory pathway: 1-22, 0.989)

$\mathrm{N}$-ter missing (secretory pathway: 1-19, 0.979 )

$\mathrm{N}$-ter missing (secretory pathway: 1-24, 0.952)

secretory pathway: 1-22, 0.986

secretory pathway : 1-19, 0.759

secretory pathway: 1-26, 0.987
At5g17920 methionine-synthase

At5g17920 methionine-synthase

At1g52030 MYROSINASE-BINDING PROTEIN 2 (MBP2 homologue)

At4g33090 peptidase M1

At3g52930 fructose-bisphosphate aldolase

At5g13420 trans-aldolase

At5g57330 aldose 1-epimerase

At5g57330 aldose 1-epimerase

At1g53240 malate dehydrogenase

At1g53240 malate dehydrogenase

At1g54040 unknown function (kelch-type beta-propeller)

At1g09010 glycoside hydrolase family 2 (GH2)

At5g20620 ubiquitin (UBQ4)

At1g31340 ubiquitin (RUB1)

At5g60390 translation elongation factor

At2g01140 fructose bisphosphate aldolase

At3g26380 unknown function (aldolase-type TIM barrel)

At5g11670 NADP-malic enzyme 2 (AtNADP-ME2 homologue)

At4g03920 nucleoside diphosphate kinase

At1g11840 glyoxalase (AtGLX1)

At2g19760 hmologous to profilin

At3g55440 triose-phosphate isomerase

At1g09310 unknown function (DUF538)

At3g04120 glyceraldehyde-3-phosphate dehydrogenase

ArthCp029 ATPase, alpha/beta subunit mitochondrion: 0.414

mitochondrion: 0.212

other: 0.860

mitochondrion: 0.408

other: 0.530

chloroplast: 0.990

other: 0.850

other: 0.836

mitochondrion: 0.700

mitochondrion: 0.789

other: 0.827

other : 0.713

$\mathrm{N}$-ter missing (other: 0.847 )

other: 0.833

other: 0.855

chloroplast : 0.828

$\mathrm{N}$-ter missing (other: 0.355 )

$\mathrm{N}$-ter missing (other: 0.963)

$\mathrm{N}$-ter missing (other: 0.383 )

other: 0.807

other: 0.805

other: 0.745

other: 0.917

$\mathrm{N}$-ter missing (other: 0.619 )

other: 0.760 
a. Protein numbers refer to Supporting information Tables S1, S2, and S3. Protein numbers in bold refer to proteins identified in the xylem sap $N$-glycoproteome.

b. Nucleotide or amino acid sequences can be found either in the CompBio (http://compbio.dfci.harvard.edu/tgi/cgi-

bin/tgi/gimain.pl?gudb=oilseed_rape) or the NCBI (http://www.ncbi.nlm.nih.gov/) dabases. Sequences are from B. napus otherwise stated.

Numbers between brackets refer to previously published xylem sap proteomes.

c. Functional domains were found as described in Material and methods. GH were annotated according to CAZy (http://www.cazy.org/) and [59].

d. Prediction of sub-cellular localization was done with TargetP (http://www.cbs.dtu.dk/services/TargetP/) and SignalP

(http://www.cbs.dtu.dk/services/SignalP/). When both predictions are consistent, only the TargetP result is shown (size of the predicted signal peptide, score). When it is not the case, both predictions are shown. When the B. oleracea protein sequence is not complete at its $\mathrm{N}$-terminus ( $\mathrm{N}$ ter), the prediction for the closest homologue in A. thaliana is shown. 


\subsection{General features of proteins identified in the $B$. oleracea xylem sap}

All the Brassica protein sequences were analyzed with bioinformatics software to predict their sub-cellular localization and the presence of functional domains. The same work was done for A. thaliana proteins homologous to Brassica sequences. The proteins could then be classified in (i) intracellular when they were devoid of signal peptide or of any signal targeting them to an intracellular compartment, and (ii) secreted proteins (Table I, Supporting information Table S3). Twenty five proteins (13\%) were predicted to be intracellular whereas 164 proteins $(87 \%)$ were predicted to be secreted. The latter proteins could be distributed in eight of the nine functional classes previously defined for $A$. thaliana cell wall proteins [29]: proteins acting on carbohydrates (29.2\%), oxido-reductases (23.8\%), proteases $(17.1 \%)$, proteins related to lipid metabolism $(4.9 \%)$, proteins involved in signaling (5.5\%), proteins with domains interacting with carbohydrates or proteins $(4.9 \%)$, miscellaneous proteins having diverse functions $(8.5 \%)$, and proteins with yet unknown function $(6.1 \%)$. No structural protein was identified in these analyses. Among the 48 proteins acting on carbohydrates, two glycoside hydrolase $(\mathrm{GH})$ families were well represented: eight proteins belonged to GH17 ( $\beta$-1,3-glucosidases), and seven to GH28 (polygalacturonases). Nearly half of the oxido-reductases were peroxidases (17), the others being proteins homologous to plastocyanins (11), multicopper oxidases (6), and berberine bridge enzymes (5). Proteases were mainly predicted as Ser proteases (12) and Asp proteases (11). Most of the proteins included in the lipid metabolism functional class were lipid transfer proteins (LTPs). Finally, all the proteins included in the signaling functional class were fasciclin AGPs (FLAs). Finding such proteins was expected because of the positive Yariv staining observed after analysis of xylem sap proteins by SDS-PAGE (Fig. 1).

All the proteins of the xylem sap proteome were also analyzed for the presence of putative $\mathrm{N}$ glycosylation sites. Proteins homologous to LTPs, ribonuclease T2, and osmotin had no predicted $N$-glycosylation sites and were only found in the xylem sap proteome. All the other proteins had predicted $\mathrm{N}$-glycosylation sites. However, 70 proteins predicted to be secreted and having $\mathrm{N}$-glycosylation sites were only found in the xylem sap proteome. One cannot exclude the possibility that they were poorly or not $\mathrm{N}$-glycosylated consistent with the fact that they were lost during the affinity chromatography step. Interestingly, although 17 out of 25 proteins predicted to be intracellular had predicted $N$-glycosylation sites, none was retained on the ConA affinity column. It suggested that the $N$-glycosylation sites of these proteins were not occupied. 
LC-MS/MS data allow getting some information on the abundance of proteins relying on spectral counting. The rationale is that the number of spectra collected for a protein in a MS/MS run is correlated to the abundance of the protein (Supporting information Tables S4). Among the proteins predicted to be intracellular, two proteins homologous to methionine synthase (proteins 9-1, 9-2) were identified with 35 and 24 spectra respectively, a protein homologous to jacalin (protein 31) with 11 spectra, and a protein homologous to peptidase M1 (protein 34) with 10 spectra. Ten out of the remaining 18 proteins predicted to be intracellular were identified with only 2 spectra which meant that they were probably present at a low level. In two independent experiments, the most abundant proteins predicted to be secreted were the following: two Ser proteases (proteins 1-1 and 12 identified with 74 and 25 spectra respectively in the reported experiment); an Asp protease (protein 18-1; 20 spectra); two $\alpha$-arabinofuranosidases (proteins 2-1 and 7-1; 43 and 29 spectra respectively); a protein homologous to peroxidase AtPrx59 (protein 11-1; 23 spectra); three berberine-bridge oxidoreductases (proteins 5, 15-1 and 15-2; 36, 23 and 20 spectra respectively); a protein homologous to AtFLA1 (protein 16-1; 20 spectra); a protein homologous to purple acid phosphatase AtPAP26 (protein 10; 24 spectra); a protein of yet unknown function with a DUF26 domain (protein 4; 32 spectra). Altogether, these results show that the proteins predicted to be intracellular are mostly minor components of the xylem sap proteome.

\subsection{Extension and screening of WalIProtDB with the $B$. oleracea xylem sap proteome}

As mentioned above, WallProtDB was built up to collect plant cell wall proteomic data [37]. It was initially devoted to A. thaliana and $O$. sativa because (i) their genomes are fully sequenced thus allowing the precise identification of proteins by MS and bioinformatics, and (ii) many proteomic studies were published on different organs. WallProtDB displays information about experimental conditions, and cell wall proteomes previously analyzed. Each protein accession number in WallProtDB has been linked to the ProtAnnDB database including bioinformatics predictions about subcellular localization, functional domains, and gene networking [36]. Literature dealing with plant cell wall proteomics has been included.

Two novelties were introduced to describe the B. oleracea xylem sap proteome and to allow comparisons with the A. thaliana cell wall proteomes characterized in different organs (Supporting information Fig. 2). First, considering the closeness of both genomes, each $B$. oleracea EST is linked to the closest $A$. thaliana gene as determined by BLASTX analysis. In many cases, two or more $B$. oleracea proteins corresponded to a single $A$. thaliana gene were 
found thanks to the presence of proteotypic peptides. This is consistent with the evolution of the Brassica genomes [42]. Second, LC-MS/MS peptide sequencing data allowing identification of $B$. oleracea proteins encoded by ESTs are provided using the X! Tandem software. The position of sequenced peptides on the translated EST or cDNA sequence can be visualized as well as MS/MS spectra and data (Supporting information Fig. 3). All this information provides a comprehensive view of the identification process using MS data.

A search was performed in WallProtDB, a cell wall proteomic database, to look for the $A$. thaliana proteins homologous to the $B$. oleracea xylem sap proteins. Eighty six proteins were already identified in at least one cell wall proteome. For example, 30 proteins were found in the leaf apoplastic proteome [18], 23 in the stem cell wall $N$-glycoproteome [19], 28 and 27 in the 5- and 11-day-old etiolated cell wall proteomes respectively [20]. Seventy eight proteins were new proteins never found in previously characterized cell wall proteomes.

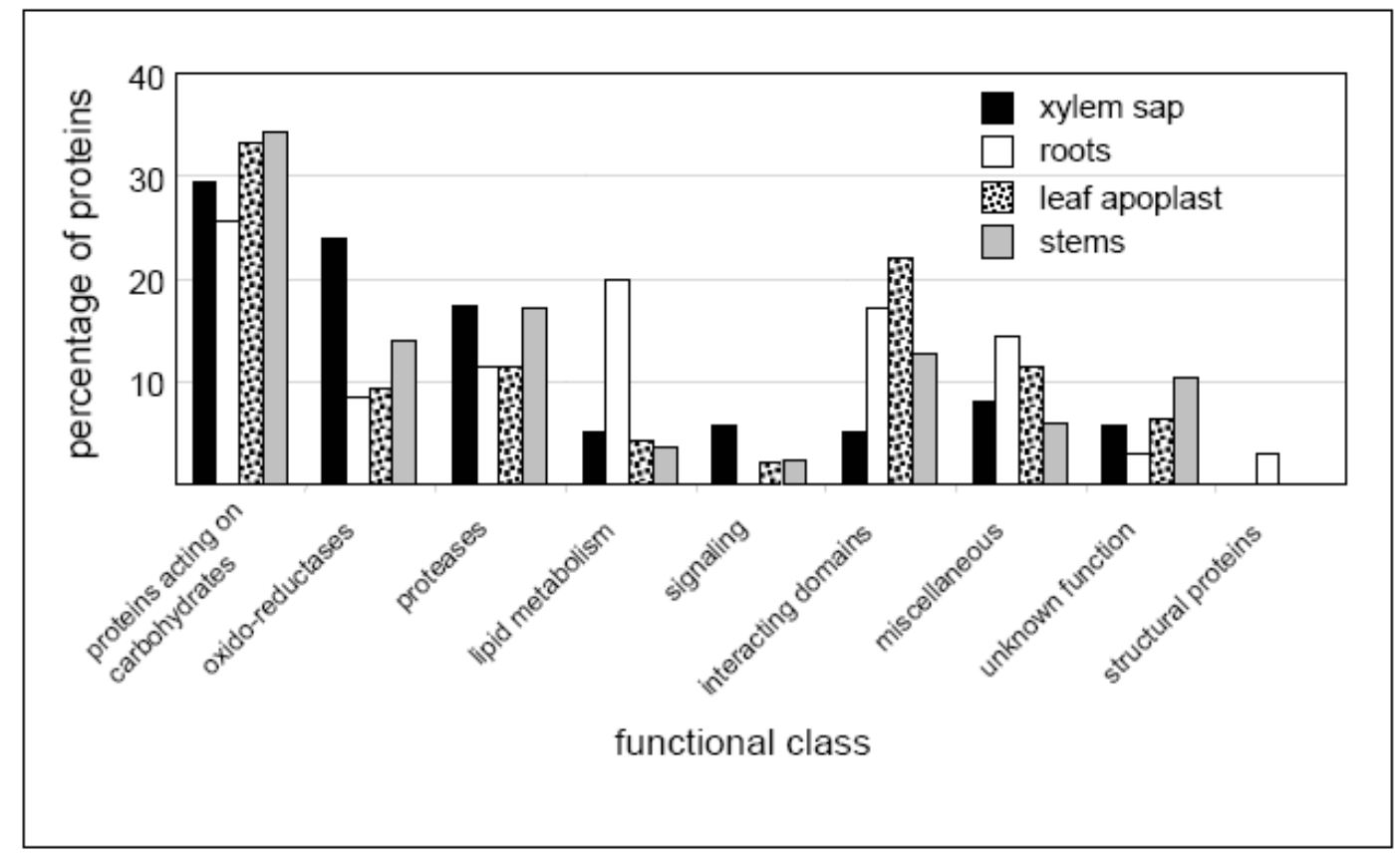

Figure 2. Comparison between the $B$. oleracea xylem sap proteome and previously characterized A. thaliana cell wall proteomes.

A. thaliana cell wall proteomes were found in the WallProtDB database (http://www.polebio.scsv.ups-tlse.fr/WallProtDB/). As in WallProtDB, B. oleracea proteins were annotated with regard to predicted functional domains and distributed in functional classes accordingly [29]. The distribution of the xylem sap proteins (xylem sap proteome, black bars) in these functional classes was compared to that of proteins previously identified in $A$. thaliana cell wall proteomes: roots (white bars) [43], rosette leaves (punctuated bars) [18], and stems (grey bars) [19]. Only proteins predicted to be secreted are considered in all cases, i.e. proteins having a predicted signal peptide and no known targeting signal in any cell compartment. 
Using WallProtDB, the whole xylem sap proteome was compared to A. thaliana cell wall proteomes of roots [43], rosette leaves [18], and stems [19]. In all cases only proteins predicted to be secreted were considered. Fig. 2 highlights several differences. There were two times more oxido-reductases in the xylem sap proteome compared to the other cell wall proteomes. The proportion of proteases was higher in the xylem sap and the stem proteomes than in the rosette leaves and root proteomes. On the contrary, there were fewer proteins with interacting domains in the xylem sap than in the other proteomes, with only two proteins homologous to lectins, two to protease inhibitors, one to xylanase inhibitors, and one to pectin methylesterase inhibitors. Structural proteins were missing as in the rosette leaves and stem proteomes.

\subsection{Expression of $\boldsymbol{A}$. thaliana genes homologous to genes encoding proteins identified in the xylem sap of $B$. oleracea in roots}

The presence of proteins in the xylem sap raises the question of the origin of these proteins. To better understand it, we looked at the pattern of expression of A. thaliana genes homologous to B. oleracea genes encoding xylem sap proteins in the AREX database which collects A. thaliana genes patterns of expression based on transcriptomics data [38]. The root is characterized by different developmental zones and tissues. We focused our attention on the root tip where xylem vessels are formed and where root absorption occurs. From outside to inside, there are different cell layers, namely epidermis, cortical cells, endodermis, pericycle and the stele comprising the xylem and the phloem vessels. Expression data could be found for $90 \%$ of the A. thaliana genes corresponding to proteins identified in the xylem sap and all those genes were found to be transcribed even at low level in root tips. Most relevant patterns were the following (Supporting information Fig. 4): expression in all root tissues except in stele parenchyma cells (pattern 1: 17\% of the genes including one third of the genes encoding proteins predicted to be intracellular); expression mostly in epidermis including or not root hairs, or cortical cells (pattern 2: 10\%); expression mostly in cortical cells, and eventually in endodermis, pericycle or stele (pattern 3: 16\%); expression in stele parenchyma stele including or not vessels (pattern 4: 37\%); expression mostly in phloem cells (pattern 5: 8\%); expression mostly in xylem cells (pattern 6: 12\%). Very few genes had transcripts neither in the stele, the pericycle or the endodermis. 


\section{Discussion}

Previous studies showed the presence of proteins in the xylem sap of different species $[4,6,8$ 17]. However, with the exception of the xylem sap proteome of an hybrid poplar [13], identification of proteins was done against heterologous sequences because of the lack of genomic or EST sequences for all the plants studied. The most complete xylem sap proteomes are those of B. napus [5], an hybrid poplar [13] and Z. mays [17] with 69, 97, and 154 proteins identified respectively. However, a detailed examination of the results indicates that several of the identified proteins show homology to different parts of the same protein and/or are identified with identical peptides. Such proteins can be present in different spots of 2D-gels or in different bands of 1D-gels, thus indicating the presence of isoforms of the same gene product as a consequence of PTMs or resulting from protein degradation. As a consequence, the number of proteins in each proteome is certainly lower. This was discussed in the case of the $Z$. mays xylem sap proteome, thus leading to the conclusion that only 59 different proteins were identified instead of 154 [17]. To our knowledge, our proteomic study provides the characterization of the largest xylem sap proteome with 189 different proteins identified. When compared to previous B. napus xylem sap proteomes [4, 7, 8], only 20 proteins out of the 189 identified in this study were already found. In addition to data obtained by MS analysis of the B. oleracea xylem sap, this study provides information on the xylem sap $\mathrm{N}$ glycoproteome. About half of the proteins ( 81 proteins) predicted to be secreted identified in the $B$. oleracea xylem sap were retained on the ConA column, showing that they are $\mathrm{N}$ glycosylated and confirming that $N$-glycosylation is a major PTM of extracellular proteins. The high proportion of secreted $N$-glycosylated proteins was expected because they pass through the endoplasmic reticulum where $N$-glycosylation occurs [44]. As expected, the thirteen proteins predicted to be secreted and devoid of $\mathrm{N}$-glycosylation sites were only found in the xylem sap proteome. For the remaining 70 proteins not retained on ConA, it is assumed that their $N$-glycans were removed in the xylem sap. Indeed all the $\mathrm{GH}$ families possibly involved in $N$-glycan degradation were found, namely $\beta$-D-xylosidases (GH3), $\mathrm{N}$-acetylhexosaminidases (GH19), $\beta$-D-galactosidases (GH35), and $\alpha$-D-mannosidases (GH38) [45]. Besides, ConA affinity chromatography was assumed to enrich the protein mixture in glycoproteins present in low amount, thus allowing to increase the coverage of the xylem sap proteome.

The proportion of proteins predicted to be intracellular (13\%) is rather low in this study. All except three of the proteins predicted to be intracellular have predicted $N$-glycosylation sites. 
However, none of them was retained on the ConA column, suggesting that their $\mathrm{N}$ glycosylation sites are not occupied. All these proteins are present at a low level apart from a protein homologous to methionine synthase. Sixty six percent of the proteins identified in hybrid poplar xylem proteome are devoid of predicted signal peptide [13]. The current hypothesis to explain the presence of intracellular proteins in xylem sap is that such proteins originate from differentiating xylem cells, and that they are released in xylem sap after cell death [13]. In the case of perennial plants such as poplar, secondary wall formation and xylem growth are more intensive than in annual plants. This would explain why there are more types of intracellular proteins in the poplar xylem sap. On the contrary, the Z. mays xylem proteome only contained proteins predicted to be secreted [17].

A major difficulty encountered in xylem sap proteome analysis relies in the harvesting step. The plants have to be decapitated and the harvesting can last for several hours. With regard to the harvesting duration, a detailed study performed in G. max showed that the 1Delectrophoresis pattern of xylem sap proteins was constant over a $28 \mathrm{~h}$-period of harvesting [10]. It suggests that major xylem sap proteins are stable xylem sap constituents and are continuously produced. With regard to the stress, it is probable that not only the cells located at the stem cut surface, but also the underground part of the plants, undergo a stress which causes changes in xylem sap composition. Many protein families identified in xylem sap proteomes could be induced by this stress, e.g. oxido-reductases, pathogenesis-related (PR)proteins, proteases and ribonucleases $([7,12,13,17]$, this study). However, there is no way to avoid this stress and all the xylem sap proteomes are obtained in similar conditions, thus allowing comparison of results.

Xylem sap proteomes appear to be very different from phloem sap proteomes. The phloem is the part of the vascular system which delivers sugars and amino acids to plant organs and carries informational molecules such as proteins, mRNAs and hormones (for a review, see [46]. Phloem protein 2 (PP2) was described as a major protein of phloem sap having RNAbinding properties or lectin activity [47]. Other proteins were identified with putative roles in defense reactions, gibberellin biosynthesis and transport [48]. The most complete phloem proteomic study identified more than 1000 proteins such as RNA-binding proteins (82 proteins) and proteins involved in protein synthesis (100 proteins) and turnover (116 proteins) [49]. Other proteins could play roles in vesicle trafficking, membrane dynamics, stress response, and redox regulation. Only one of the 45 most abundant proteins, a peroxidase 
homologous to At5g07630, was predicted to be secreted. Altogether, the xylem and phloem sap proteomes thus appear to be very different, as expected from their different physiological roles.

All the proteomic data were included in WallProtDB, a database dedicated to cell wall proteomics. WallProtDB is a tool complementary to existing databases since it allows direct comparison between cell wall proteomes of various organs of A. thaliana and O. sativa. Only data from plants with genomes completely sequenced or large collection of ESTs are included since unequivocal identification of proteins by peptide mass fingerprinting or peptide sequencing can be done. Other proteomic databases such as the Plant Proteome Database (PPDB, http://ppdb.tc.cornell.edu/) and the Atproteome database (http://fgczatproteome.unizh.ch/) are built in a different way. For each gene, the latter databases give information on the conditions in which the proteins were identified as well as MS data when available. Being devoted to cell wall proteomes only, WallProtDB resembles AT_CHLORO (http://www.grenoble.prabi.fr/at_chloro/) which describes the chloroplast proteome. In this new version, MS data are also included for the B. oleracea xylem sap proteome. The next step would be to crosslink all the plant proteomic databases to get all the information at the same place as was done in the Human Proteomic reference database (http://www.hprd.org/). It would give the plant community a great tool to better understand protein structure and gene regulation.

An interesting outcome of the new version of WallProtDB is the comparison between the $B$. oleracea xylem sap proteome and previously characterized cell wall proteomes of A. thaliana. Indeed, the fact that xylem sap is considered as part of the apoplast and the closeness of the two species allowed this comparison. Three features distinguish the B. oleracea proteome from previously characterized cell wall proteomes [29]: there are more oxido-reductases, more proteases, and less enzyme inhibitors. As discussed above, because of the technical constrains to collect xylem sap, we cannot exclude that some of the proteins related to stress response may change during the harvesting period. Among oxido-reductases, peroxidases represent one tenth of the predicted secreted proteins identified. Peroxidases were previously described as important proteins in xylem sap proteomes. They were associated either to lignin biosynthesis in xylem vessels undergoing differentiation or to plant defense $[4,7,12,13]$. Proteins homologous to multicopper oxidases were also found to be numerous in A. thaliana stems at late flowering stage when lignification occurs [19]. SKU5 (SKEWED 5) was shown 
to be involved in root growth and SKS6 (SKU5-SIMILAR 6) to contribute to cotyledon vascular patterning $[50,51]$. Blue copper binding proteins were abundant in A. thaliana cell suspension cultures. Although their exact role in cell walls is not known, they have been associated to redox processes as electron transfer proteins with small molecular weight compounds [52]. A great proportion of proteases is the second feature of the B. oleracea xylem sap proteome. It is the first time that so many proteases are identified. Different specificities could be predicted such as Ser proteases (subtilases), Ser carboxypeptidases, Cys proteases, and Asp proteases. Proteases are assumed to play roles in maturation of enzymes, signaling, protein turnover, and defense against pathogens [53]. It was previously shown that maturation of enzymes occur in the cell wall [54]. Over-expression of CDR1 (CONSTITUTIVE DISEASE RESISTANT 1) encoding an A. thaliana Asp protease causes dwarfism and resistance to virulent Pseudomonas syringae [55]. Finally, in all previously characterized plant extracellular proteomes, enzymes and the corresponding inhibitors are present, probably allowing fine regulation of enzymatic activities [20]. In the B. oleracea xylem sap, there are only a few enzyme inhibitors (two protease inhibitors, a pectin methyl esterase inhibitor, and a xylanase inhibitor). It suggests that the enzymes are fully active. Some of them may play roles in defense reactions against pathogens invading the xylem vessels.

Three additional protein functional classes deserve comments. The main one comprises proteins acting on carbohydrates. Forty two proteins having GH domains were identified. Several of them could play roles in hydrolysis of PTMs of $\mathrm{N}$-glycoproteins as discussed above. Others are assumed to contribute to defense reactions, such as $\beta$-1,3-glucanases (GH17, 6 proteins) and chitinases/lyzozymes (GH18-19, 5 proteins) [45]. More puzzling are the roles of polygalacturonases and $\alpha$-L-arabinofuranosidases/ $\beta$-D-xylosidases. Polygalacturonases (GH28, 7 proteins) are assumed to play roles in the organization of pectins and in their modification in response to pathogen attack [45]. However, the contribution of pectins to secondary walls is very low. $\alpha$-L-arabinofuranosidases/ $\beta$-Dxylosidases (GH51, 4 proteins) are major proteins in xylem sap as estimated from MS data. Such proteins were also identified in the poplar xylem sap [13]. Their preferred substrates in cell wall are assumed to be arabinoxylan, and arabinan as inferred from in vitro tests [56]. The second functional class to be mentioned is that of proteins possibly involved in signaling. It is the first time the importance of FLAs in xylem sap can be stressed. Indeed, seven proteins homologous to A. thaliana FLAs (AtFLA1, 2, 7-10) were identified. Their roles in cell walls 
are not yet understood, but they were found to accumulate at the inner side of the G-layer of the xylem of poplar tension wood. They were assumed to have a specific function in the building of this cell wall layer [57]. Finally no structural protein could be identified in the $B$. oleracea xylem sap although a glycine-rich protein (GRP) was previously found in the $B$. napus xylem sap proteome [4]. Such proteins were shown to be present in cucumber xylem sap and to accumulate in the walls of cucumber root metaxylem cells [6]. A bean GRP was shown to be synthesized by living protoxylem cells and xylem parenchyma cells, and to be transported from xylem parenchyma cells to the protoxylem wall after cell death [58].

Several authors have already discussed the origin of xylem sap proteins [3, 10, 12]. In the root, the endodermis constitutes a barrier preventing the movement of organic substances and even water from the epidermis and cortical cells to the stele through the apoplast. It is assumed that proteins present in the xylem sap are synthesized in the stele cells. They would then be delivered apoplastically to the xylem sap thus considered as part of the apoplast. In this study, we looked at the pattern of expression of the A. thaliana genes homologous to the B. oleracea xylem sap proteins using the AREX database. All the genes were found to be expressed in the root tip, and only a few of them were expressed neither in the stele, the pericycle, or the endodermis. It suggests that the proteins were synthesized in the root tip, and then loaded into the xylem sap. These data are consistent with previous experimental data. A cucumber GRP was shown to be synthesized in the vascular tissues of the root and assumed to be transported over long distance via the xylem sap to vessels and sclerenchyma of aboveground organs [6]. Xylem sap proteins as diverse as a cucumber lectin [5], a cucumber peroxidase [12], and a tomato Cys-rich protein with structural similarity to LTPs [14] were found in roots and the genes encoding the lectin and the Cys-rich protein were shown to be transcribed in roots. Altogether, these data strengthen the hypothesis of the production of xylem sap proteins in the root stele, and their further loading into the xylem sap. The water flow would then ensure their long distance transport to aboveground organs. The composition of the xylem sap proteome suggests roles in xylem differentiation and in plant defense. However, additional investigations are required to better understand the function of xylem sap proteins. 
The authors are thankful to Université Paul Sabatier (Toulouse, France), CNRS and INRA for supporting their research work. Financial support was provided by the French Agence Nationale de la Recherche (Grant ANR-08-BLAN-0193-01). LC-MS/MS analyses were performed on the Plateforme d'Analyse Protéomique de Paris Sud-Ouest (PAPPSO). The authors also wish to thank Thibaut Douché, Dr Catherine Digonnet, and Pr Christophe Dunand for stimulating discussions.

The authors have declared no conflict of interest.

\section{References}

[1] Sattelmacher, B., The apoplast and its significance for plant mineral nutrition. New Phytol. 2001, 149, 167-192.

[2] Fukuda, H., Xylogenesis: initiation, progression, and cell death. Annu Rev Plant Physiol Plant Mol Biol 1996, 47, 299-325.

[3] Satoh, S., Organic substances in xylem sap delivered to above-ground organs by the roots. J Plant Res 2006, 119, 179-187.

[4] Kehr, J., Buhtz, A., Giavalisco, P., Analysis of xylem sap proteins from Brassica napus. BMC Plant Biology 2005, 5, 11.

[5] Masuda, S., Sakuta, C., Satoh, S., cDNA cloning of a novel lectin-like xylem sap protein and its root-specific expression in cucumber. Plant Cell Physiol 1999, 40, 1177-1181.

[6] Sakuta, C., Satoh, S., Vascular tissue-specific gene expression of xylem sap glycinerich proteins in root and their localization in the walls of metaxylem vessels in cucumber. Plant Cell Physiol 2000, 41, 627-638.

[7] Buhtz, A., Kolasa, A., Arlt, K., Walz, C. et al., Xylem sap protein composition is conserved among different plant species. Planta 2004, 219, 610-618.

[8] Floerl, S., Druebert, C., Majcherczyk, A., Karlovsky, P. et al., Defence reactions in the apoplastic proteome of oilseed rape (Brassica napus var. napus) attenuate Verticillium longisporum growth but not disease symptoms. BMC Plant Biology 2008, 8, 129.

[9] Oda, A., Sakuta, C., Masuda, S., Mizoguchi, T. et al., Possible involvement of leaf gibberellins in the clock-controlled expression of XSP30, a gene encoding a xylem sap lectin, in cucumber roots. Plant Physiol 2003, 133, 1779-1790.

[10] Djordjevic, M., Oakes, M., Li, D., Hwang, C. et al., The Glycine max xylem sap and apoplastic proteome. J Proteome Res 2007, 6, 3771-3779.

[11] Subramanian, S., Cho, U.-H., Keyes, C., Yu, O., Distinct changes in soybean xylem sap proteome in response to pathogenic and symbiotic microbe interactions. $B M C$ Plant Biol 2009, 9, 119.

[12] Biles, C., Abeles, F., Xylem sap proteins. Plant Physiol 1991, 96, 597-601.

[13] Dafoe, N., Constabel, P., Proteomic analysis of hybrid poplar xylem sap. Phytochemistry 2009, 70, 856-863.

[14] Rep, M., Dekker, H., Vossen, J., de Boer, A. et al., A tomato xylem sap protein represents a new family of small cysteine-rich proteins with structural similarity to lipid transfer proteins. FEBS Lett 2003, 534, 82-86.

[15] Rep, M., Dekker, H., Vossen, J., de Boer, A. et al., Mass spectrometric identification of isoforms of PR proteins in xylem sap of fungus-infected tomato. Plant Physiol 2002, 130, 904-917. 
[16] Houterman, P., Speijer, D., Dekker, H., de Koster, C. et al., The mixed xylem sap proteome of Fusarium oxysporum-infected tomato plants. Molec Plant Pathol 2007, 8, 215-221.

[17] Alvarez, S., Goodger, J. Q., Marsh, E. L., Chen, S. et al., Characterization of the maize xylem sap proteome. J. Proteome Res. 2006, 5, 963-72.

[18] Boudart, G., Jamet, E., Rossignol, M., Lafitte, C. et al., Cell wall proteins in apoplastic fluids of Arabidopsis thaliana rosettes: Identification by mass spectrometry and bioinformatics. Proteomics 2005, 5, 212-221.

[19] Minic, Z., Jamet, E., Negroni, L., der Garabedian, P. A. et al., A sub-proteome of Arabidopsis thaliana trapped on Concanavalin A is enriched in cell wall glycoside hydrolases. J. Exp. Bot. 2007, 58, 2503-2512.

[20] Irshad, M., Canut, H., Borderies, G., Pont-Lezica, R. et al., A new picture of cell wall protein dynamics in elongating cells of Arabidopsis thaliana: Confirmed actors and newcomers. BMC Plant Biol. 2008, 8, 94.

[21] Charmont, S., Jamet, E., Pont-Lezica, R., Canut, H., Proteomic analysis of secreted proteins from Arabidopsis thaliana seedlings: improved recovery following removal of phenolic compounds. Phytochemistry 2005, 66, 453-461.

[22] Bayer, E. M., Bottrill, A. R., Walshaw, J., Vigouroux, M. et al., Arabidopsis cell wall proteome defined using multidimensional protein identification technology.

Proteomics 2006, 6, 301-11.

[23] Schultz, C. J., Ferguson, K. L., Lahnstein, J., Bacic, A., Post-translational modifications of arabinogalactan-peptides of Arabidopsis thaliana. J. Biol. Chem. 2004, 279, 455103-45511.

[24] Borderies, G., Jamet, E., Lafitte, C., Rossignol, M. et al., Proteomics of loosely bound cell wall proteins of Arabidopsis thaliana cell suspension cultures: A critical analysis. Electrophoresis 2003, 24, 3421-3432.

[25] Chivasa, S., Ndimba, B. K., Simon, W. J., Robertson, D. et al., Proteomic analysis of the Arabidopsis thaliana cell wall. Electrophoresis 2002, 23, 1754-1765.

[26] Kwon, H.-K., Yokoyama, R., Nishitani, K., A proteomic approach to apoplastic proteins involved in cell wall regeneration in protoplasts of Arabidopsis suspensioncultured cells. Plant Cell Physiol. 2005, 46, 843-857.

[27] Robertson, D., Mitchell, G. P., Gilroy, J. S., Gerrish, C. et al., Differential extraction and protein sequencing reveals major differences in patterns of primary cell wall proteins from plants. J. Biol. Chem. 1997, 272, 15841-15848.

[28] Prakash, S., Bhat, S., Quiros, C., Kirti, P. et al., in J. Janick (Ed.), Plant Breeding Reviews, vol 31, John Wiley \& Sons, Inc, Hoboken, NJ, 2009, pp. 21-188.

[29] Jamet, E., Albenne, C., Boudart, G., Irshad, M. et al., Recent advances in plant cell wall proteomics. Proteomics 2008, 8, 893-908.

[30] Kehr, J., Rep, M., Protein extraction from xylem and phloem sap. Methods Mol Biol 2007, 355, 27-35.

[31] Laemmli, U. K., Cleavage of the structural proteins during the assembly of the head of bacteriophage T4. Nature 1970, 227, 680-685.

[32] Scheler, C., Lamer, S., Pan, Z., Li, X. P. et al., Peptide mass fingerprinting sequences coverage from differentially stained proteins in two-dimensional electrophoresis patterns by matrix assisted laser desorption/ionization-mass spectrometry (MALDIMS). Electrophoresis 1998, 19, 918-927.

[33] Willats, W. G., Knox, J. P., A role for arabinogalactan-proteins in plant cell expansion: evidence from studies on the interaction of $\beta$-glucosyl Yariv reagent with seedlings of Arabidopsis thaliana. Plant J. 1996, 9, 919-925. 
[34] Martin, A., J, L., Kichey, T., Gerentes, D. et al., Two cytosolic glutamine synthetase isoforms of maize are specifically involved in the control of grain production. Plant Cell 2006, 18, 3252-3274.

[35] Frottin, F., Espagne, C., Traverso, J., Mauve, C. et al., Cotranslational proteolysis dominates glutathione homeostasis to support proper growth and development. Plant Cell 2009, 21, 3296-3314.

[36] San Clemente, H., Pont-Lezica, R., Jamet, E., Bioinformatics as a tool for assessing the quality of sub-cellular proteomic strategies and inferring functions of proteins: plant cell wall proteomics as a test case. Bioinform. Biol. Insights 2009, 3, 15-28.

[37] Pont-Lezica, R., Minic, Z., San Clemente, H., Roujol, D. et al., in M. A. Osborne (Ed.), Advances in Genetics Research, Nova Science Publishers, Inc., Hauppauge, NY, 2010.

[38] Birnbaum, K., Shasha, D., Wang, J., Jung, J. et al., A gene expression map of the Arabidopsis root. Science 2003, 302, 801-806.

[39] Altschul, S. F., Gish, W., Miller, W., Myers, E. W. et al., Basic local alignement search tool. J. Mol. Biol. 1990, 215, 403-410.

[40] Bradford, M. M., A rapid and sensitive method for the quantitation of microgram quantities of protein utilizing the principle of protein-dye binding. Anal. Biochem. 1976, 72, 248-254.

[41] Jamet, E., Canut, H., Boudart, G., Pont-Lezica, R., Cell wall proteins: a new insight through proteomics. Trends Plant Sci. 2006, 11, 33-39.

[42] Lagerkrantz, U., Comparative mapping between Arabidopsis thaliana and Brassica nigra indicates that Brassica genomes have evolved through extensive genome replication accompanied by chromosome fusions and frequent rearrangements. Genetics 1998, 150, 1217-1228.

[43] Basu, U., Francis, J. L., Whittal, R. W., Stephens, J. L. et al., Extracellular proteomes of Arabidopsis thaliana and Brassica napus roots: analysis and comparison by MUdPIT and LC-MS/MS. Plant Soil 2006, 286, 357-376.

[44] Faye, L., Boulaflous, A., Benchabane, M., Gomord, V. et al., Protein modifications in the plant secretory pathway: current status and practical implications in molecular pharming. Vaccine 2005, 23, 1770-1778.

[45] Minic, Z., Physiological roles of plant glycoside hydrolases. Planta 2008, 227, 723740.

[46] Thompson, G., Schulz, A., Macromolecular trafficking in the phloem. Trends Plant Sci 1999, 4, 354-360.

[47] Dinant, S., Clark, A., Zhu, Y., Vilaine, F. et al., Diversity of the superfamily of phloem lectins (Phloem Protein 2) in angiosperms. Plant Physiol 2003, 131, 114-128.

[48] Cho, W., Chen, X.-Y., Rim, Y., Chu, H. et al., Proteome study of the phloem sap of pumpkin using multidimensional protein identification technology. J Plant Physiol 2010, 167, 771-778.

[49] Lin, M.-K., Lee, Y.-J., Lough, T., Phinney, B. et al., Analysis of the pumpkin phloem proteome provides insight into angiosperm sieve tube function. Mol Cell Proteomics 2009, 8, 343-356.

[50] Jacobs, J., Roe, J. L., SKS6, a multicopper oxidase-like gene, participates in cotyledon vascular patterning during Arabidopsis thaliana development. Planta 2005, 222, 652666.

[51] Sedbrook, J. C., Carroll, K. L., Hung, K. F., Masson, P. H. et al., The Arabidopsis SKU5 gene encodes an extracellular glycosyl phosphatidylinositol-anchored glycoprotein involved in directional root growth. Plant Cell 2002, 14, 1635-1648. 
[52] Nersissian, A. M., Shipp, E. L., Blue copper-binding domains. Adv. Protein Chem. 2002, 60, 271-340.

[53] Schaller, A., A cut above the rest: the regulatory function of plant proteases. Planta 2004, 220, 183-97.

[54] Albenne, C., Canut H, Boudart, G., Zhang, Y. et al., Plant cell wall proteomics: mass spectrometry data, a trove for research on protein structure/function relationships. Mol. Plant 2009, 2, 977-989.

[55] Xia, Y., Suzuki, H., Borevitz, J., Blount, J. et al., An extracellular aspartic protease functions in Arabidopsis disease resistance signaling. EMBO J 2004, 23, 980-8.

[56] Minic, Z., Rihouey, C., Do, C., Lerouge, P. et al., Purification and characterization of enzymes exhibiting beta-D-xylosidase activities in stem tissues of Arabidopsis. Plant Physiol 2004, 135, 867-878.

[57] Lafarguette, F., Leple, J. C., Dejardin, A., Laurans, F. et al., Poplar genes encoding fasciclin-like arabinogalactan proteins are highly expressed in tension wood. New Phytol. 2004, 164, 107-121.

[58] Ringli, C., Keller, B., Ryser, U., Glycine-rich proteins as structural components of plant cell walls. Cell. Mol. Life Sci. 2001, 58, 1430-41.

[59] Minic, Z., Jouanin, L., Plant glycoside hydrolases involved in cell wall polysaccharide degradation. Plant Physiol Biochem 2006, 44, 435-449. 


\section{Supporting information}

Supporting information Table S1. Identification by LC-MS/MS of proteins present in the $B$. oleracea xylem sap (xylem sap proteome).

Supporting information Table S2. Identification by LC-MS/MS of proteins of $B$. oleracea retained by ConA affinity chromatography (xylem sap $N$-glycoproteome).

Supporting information Table S3. Description of the xylem sap proteome and of the xylem sap $N$-glycoproteome of B. oleracea.

Supporting information Table S4. Spectral counting data for the most abundant proteins identified in the B. oleracea xylem sap proteome and for the proteins predicted to be intracellular.

Supporting information Fig. 1. Xylem sap sampling from B. oleracea cut stems.

Supporting information Fig. 2. Content of WallProtDB.

Supporting information Fig. 3. LC-MS/MS data as shown in WallProtDB.

Supporting information Fig. 4. Root pattern of expression of $A$. thaliana genes encoding proteins homologous to $B$. oleracea xylem sap proteins. 